\title{
Parallelism: the foundation of biomarker assay development and validation
}

\author{
Devangi Mehta1, Shobha Purushothama ${ }^{1}$ \& Lauren Stevenson*,1,2 \\ ${ }^{1}$ Development Biomarkers \& Bioanalytical Sciences, Biogen, Inc., 225 Binney Street, Cambridge, MA 02142, USA \\ ${ }^{2}$ Address \\ *Author for correspondence: lauren.stevenson@biogen.com
}

First draft submitted: 20 December 2017; Accepted for publication: 22 January 2018; Published online: 20 June 2018

Keywords: dilutional linearity • minimum required dilution • parallelism • selectivity

In recent years, there has been growing appreciation that biomarker assay development and validation must be considered in their own context and not incongruously fitted into concepts/approaches developed to evaluate PK assay performance. This opinion, succinctly coined as 'Biomarker assays are not PK assays' in the LBA sessions at the Crystal City VI Workshop on bioanalytical method validation for biomarkers $[1,2]$ has since become the basis for driving discussions on the appropriate scientific approaches for biomarker assay development and validation, with characterization of parallelism being a foundational component. Over the last ten years, there has been an increasing amount of literature in the ligand-binding assay field discussing the relative importance of evaluating parallelism in the context of both pharmacokinetic and biomarker assay development and validation [3-6]. Importantly, in June 2017 a public workshop entitled 'Regulatory considerations for the analytical validation of assays used in the qualification of biomarkers in biological matrices' was held in Washington DC, USA with the goal of creating alignment on the evidentiary considerations for the analytical validation of biomarker assays within their specific context(s) of use [7]. Discussions at that workshop highlighted that there remain gaps in both breadth and depth of conceptual understanding of parallelism among professionals across industry, academia and regulatory agencies. Here, we summarize the critical concepts associated with parallelism assessments in LBA biomarker assay development and validation, including key discussion points raised at the public workshop.

Parallelism is fundamental to LBA biomarker assay development and subsequent validation. The classical purpose of parallelism is to demonstrate that the sample-dilution response curve is parallel to the standard-calibrator response curve, thus confirming that the calibrator material is suitable for measurement of the endogenous analyte $[5,8,9]$. Indeed, when multiple individual samples demonstrate parallel responses compared with one another but not with the calibrator, the quality of the calibrator material should be questioned and other sources of calibrator evaluated [6]. In addition to this essential goal, the parallelism assessment informs numerous other assay parameters, including selection of the surrogate matrix/assay buffer, establishment of the assay's minimum required dilution (MRD), evaluation of selectivity and determination of the LLOQ with respect to the endogenous analyte. To note, many of these assessments can be performed simultaneously within a single parallelism experiment.

Generally speaking, successful demonstration of parallelism indicates that the selected surrogate matrix is appropriate for the assay. Indeed, a parallelism assessment can be leveraged early in assay development as a means to select an appropriate surrogate matrix. In a recent publication, Tu and Bennett proposed a 'raw signal' parallelism approach that enables the simultaneous evaluation of multiple potential surrogate matrix buffers as an efficient means to select surrogate matrix [6].

In order to then determine assay MRD, parallelism results from multiple individual samples are examined to identify the dilution at which matrix effects are ameliorated and all samples return results within assay range. Concentrations measured at this provisional MRD can then be set as the reference nominal concentrations against which concentration results from subsequent dilutions are compared. This exercise may require an iterative approach, whereby different provisional MRDs (and their associated reference nominal concentrations) are evaluated. The minimum dilution at which acceptable relative accuracy (or bias) is achieved from multiple subsequent dilutions for each of the individual samples tested can be considered the assay's MRD [5]. The same experiment may also 
be considered a selectivity assessment, since demonstration of parallelism across multiple individuals effectively establishes that the endogenous analyte is being selectively measured in the milieu of complex matrix components at the identified MRD up to the maximum acceptable dilution.

Since the calibrator material used in biomarker assays often differs from the endogenous analyte and interaction with the critical reagents may differ to a greater or lesser degree, biomarker assay sensitivity, or LLOQ should be determined in the context of the endogenous analyte via parallelism. For this analysis, multiple individual samples are diluted to below the assay's detection limit (as defined by the standard curve) and each sample dilution series is examined to identify the dilution range that results in a parallel response for each sample. We have previously proposed that the LLOQ may then be set using two different approaches, the common dilution method and the common concentration method [5]. Although both methods may be applied in a fit-for-purpose manner, we typically apply the common-concentration method for biomarker assays implemented beyond the exploratory phase. Using this method, all individual dilution series are examined and the lowest concentration for each sample that provides a parallel response is noted. The highest concentration from that set of samples is then set as the LLOQ as this represents a concentration at which all individual samples demonstrated parallelism.

Practically speaking, each of the above assay parameters can be assessed within a single experiment, but this does require samples that possess adequate levels of endogenous analyte. Unsurprisingly, perhaps the most common question that arises during scientific discussions on parallelism is, "What if samples with adequate levels of endogenous analyte are not available?" This question received much attention at the public workshop in June where the merits of alternative approaches were discussed at length. These approaches included production of' endogenous' analyte (e.g., production of cytokines through cell stimulation) which can then be spiked into matrix for parallelism assessments and spike recovery of recombinant/purified material on top of endogenous analyte. Both approaches are ultimately imperfect intermediate solutions and should only be considered when genuine endogenous samples are impossible to source. Once, samples with appropriate endogenous levels become available (typically in-study), then parallelism should be revisited and assay performance claims confirmed or revised accordingly.

Several concerns related to the validity of spiking approaches were highlighted by industry representatives at the public workshop. It was emphasized that any results obtained from spike-recovery or spike-dilution experiments should be interpreted with caution. Although it may be encouraging, if such an approach works, providing some relative confidence that the critical reagents see endogenous and recombinant/purified material similarly, one should beware a false sense of security [10]. If only samples with very low levels of endogenous analyte are available, spiking a large concentration of recombinant calibrator on top of the endogenous analyte and performing a recovery experiment or dilution study will be uninformative with respect to detection of the endogenous analyte. For example, a starting sample with $10 \%$ endogenous analyte and $90 \%$ recombinant material will reliably pass both evaluations even if the assay measures only the recombinant material. In the event that the balance of endogenous and spiked recombinant material is more equivalent, there should be scope for evaluation of the endogenous alone, even if only at a few dilutions. Additional data from samples spiked with ever higher levels of recombinant material will only provide information on measurement of the recombinant material, which is irrelevant to addressing the assay's suitability to measure the endogenous analyte of interest. Conversely, if spiking experiments do not work, this does not categorically demonstrate that the assay is not viable. Such results may be obtained when endogenous analyte exists as a multimer while recombinant material as a free monomer.

Importantly, a parallelism failure may or may not negate the use of the assay, depending upon the context of use. Either way, parallelism data can be leveraged to inform how the current assay and the data resulting from it may be used, as well as provide insights into the nature of the endogenous analyte and guide assay redevelopment efforts. Therefore, since only parallelism assessments address measurement of endogenous analyte, alternative approaches should only serve as imperfect stop gaps until parallelism can be properly addressed.

The ongoing dialogue on biomarker assay development and validation has been productive and encouraging, with real progress being made. Beyond the topics discussed herein, several outstanding questions remain. The scientific community will need to continue to align and refine thinking on how to set nominal concentrations and appropriate acceptance criteria for parallelism assessments based on the biomarker's context of use. In addition, at present we do not have approaches to meaningfully evaluate LBA biomarker assay specificity. Although, it is widely recognized that characterization of critical reagents plays an important role in understanding specificity, recommendations for what constitutes adequate and appropriate reagent characterization need to be developed. Despite these challenges, the progress we have made in evolving a more biologically relevant approach to evaluating LBA biomarker assays, including recognition of parallelism as the foundation of biomarker assay development 
and validation, is reason for optimism. Continuing along this path will lead to assays that generate higher quality data and enable biomarkers to be more effectively leveraged in clinical development and the real-world setting, ultimately offering better outcomes for patients.

\section{Open access}

This work is licensed under the Attribution-NonCommercial-NoDerivatives 4.0 Unported License. To view a copy of this license, visit http://creativecommons.org/licenses/by-nc-nd/4.0

\section{Financial \& competing interests disclosure}

The authors have no relevant affiliations or financial involvement with any organization or entity with a financial interest in or financial conflict with the subject matter or materials discussed in the manuscript. This includes employment, consultancies, honoraria, stock ownership or options, expert testimony, grants or patents received or pending, or royalties.

No writing assistance was utilized in the production of this manuscript.

\section{References}

1. Arnold ME, Booth B, King L, Ray C. Workshop report: crystal city VI - bioanalytical method validation for biomarkers. AAPS J. 18(6), 1366-1372 (2016).

2. Stevenson LF. Challenges in making LBA and LC/MS biomarker assays meet PK assay criteria: LBA concepts. Presented at: AAPS Crystal City VI Workshop: Bioanalytical Method Validation for Biomarkers. MD, USA, 28-29 September 2015.

3. Lee JW, Devanarayan V, Barrett YC et al. Fit-for-purpose method development and validation for successful biomarker measurement. Pharm. Res. 23(2), 312-328 (2006).

4. Valentin MA, Ma S, Zhao A, Legay F, Avrameas A. Validation of immunoassay for protein biomarkers: bioanalytical study plan implementation to support pre-clinical and clinical studies. J. Pharm. Biomed. Anal. 55(5), 869-877 (2011).

5. Stevenson LF, Purushothama S. Parallelism: considerations for the development, validation and implementation of PK and biomarker ligand-binding assays. Bioanalysis 6(2), 185-198 (2014).

6. Tu J, Bennett P. Parallelism experiments to evaluate matrix effects, selectivity and sensitivity in ligand-binding assay method development: pros and cons. Bioanalysis 9(14), 1107-1122 (2017).

7. Duke-Margolis Center for Health Policy Public workshop: scientific and regulatory considerations for the analytical validation of assays used in the qualification of biomarkers in biological Matrices. DC, USA, 14-15 June 2017. https://healthpolicy.duke.edu/events/public-workshop-scientific-and-regulatory-considerations-analytical-validation-assays-used

8. King LE. Parallelism experiments in biomarker ligand-binding assays to assess immunological similarity. Bioanalysis 8(23), 2387-2391 (2016).

9. Cowan KJ. Implementing fit-for-purpose biomarker assay approaches: a bioanalytical perspective. Bioanalysis 8(12), 1221-1223 (2016).

10. Timmerman P, Scheel Fjording M, Allinson J et al. Feedback from the EBF-Focus workshop: bringing assay validation and analysis of biomarkers into practice. Bioanalysis 9(9), 675-681 (2017). 
